The Astrophysical JouRnal, 555:775-785, 2001 July 10

(C) 2001. The American Astronomical Society. All rights reserved. Printed in U.S.A.

\title{
CHARACTERISTIC ULTRAVIOLET/OPTICAL TIMESCALES IN ACTIVE GALACTIC NUCLEI
}

\author{
Stefan Collier $^{1}$ and Bradley M. Peterson ${ }^{1}$ \\ Received 2000 November 1; accepted 2001 March 20
}

\begin{abstract}
The UV/optical light curves of active galactic nuclei (AGNs) may be roughly characterized by aperiodic fractional flux variations of $\sim 10 \%$ on timescales of 1 month. The physical mechanism(s) responsible remain ill defined. We present a structure function analysis, i.e., measure the power distribution over a range of timescales $\tau$, of 13 AGNs to constrain the origin of UV/optical emission. On timescales $\tau \sim 5-$ 60 days, the mean UV and optical power-density spectra (PDS) are equivalent. This may suggest that the underlying energy generating mechanism is identical. The combined UV/optical PDS is $P(f) \propto f^{-\alpha}$ with $\alpha=2.13_{-0.06}^{+0.22}$. For sources with measured X-ray PDS indices, we find they are indistinguishable from their UV/optical counterparts. This supports scenarios whereby X-rays are generated via Compton upscattering of UV photons, to later radiatively drive optical variations. At the same time, we present evidence for characteristic variability timescales $\tau_{\text {char }}$ of $\sim 5-100$ days in 10 sources. These variability timescales combined with reverberation based masses $M$ suggest a $M-\tau_{\text {char }}$ relationship; higher mass systems have larger characteristic timescales. The UV $\tau_{\text {char }}$ may possibly reflect dynamical or accretion disk thermal timescales. We find suggestive evidence for a dichotomy, at $\tau \sim 30$ days and $M \sim 10^{7} M_{\odot}$, between short- and long-timescale optical variations. These optical variations may be attributable to dynamical and accretion disk thermal or starburst activity timescales, respectively.

Subject headings: accretion, accretion disks - galaxies: active - ultraviolet: galaxies $-\mathrm{X}$-rays: galaxies
\end{abstract}

\section{INTRODUCTION}

The nature of the UV/optical emission is an important issue, since it dominates the bolometric luminosity of radioquiet active galactic nuclei (AGNs). Its origin defines the primary energy generating mechanism and thus has important ramifications for understanding their central engines. The observed quasi-simultaneity, to within $\approx$ days, of UV/ optical variations in six Seyfert 1 galaxies (Peterson et al. 1998, and references therein) suggests the variations are radiatively driven by higher energy variations (e.g., George \& Fabian 1991), i.e., a disk reprocessing scenario. Other timescales associated with the propagation of variations, e.g., thermal disk instabilities, are of order months to years. Additional observations show that the detailed relationship between multiwavelength variations is complex. The identification of the driving high-energy radiation is poorly defined (e.g., Nandra et al. 2000; Marshall et al. 1997), most likely because it falls in the unobservable EUV/FUV energy range. Furthermore, the hard X-ray and optical variations exhibit variable and complex behavior on different timescales and provide severe challenges to simple reprocessing models (e.g., Edelson et al. 2000; Nandra et al. 1998). It is clear, however, that future accurate definition of the relationship between multicolor variations will strongly constrain the sizes and origin of the continuum emitting sources.

A complementary approach for discerning the energy generation mechanisms is by measuring the fluctuation power-density spectrum (PDS) of the light curve and comparing it to those predicted by, e.g., starburst (Terlevich et al. 1992, 1995; Aretxaga \& Terlevich 1994) and disk instability models (e.g., "bright-spot" model of Abramowicz et al. 1991; "self-organized critical" [SOC] model of Mineshige, Takeuchi, \& Nishimori 1994), i.e., $P(f) \propto f^{-\alpha}$ with

\footnotetext{
${ }^{1}$ Department of Astronomy, Ohio State University, 140 West 18th Avenue, Columbus, OH 43210; stefan@astronomy.ohio-state.edu, peterson@astronomy.ohio-state.edu.
}

$\alpha \sim 2.6$ and 1.9, respectively (Kawaguchi et al. 1998). Moreover, the measurement of a characteristic variability timescale $\tau_{\text {char }}$, defined loosely by the point at which the power spectrum begins to turn over, may permit determination of the source size and black hole mass. In starburst models the luminosity variations are caused by supernova explosions and subsequent evolution of their compact remnants (cSNRs), with $\tau_{\text {char }}$ possibly related to the lifetime of the cSNRs. For disk instability models the variability is due to, e.g., an ensemble of random, magnetic reconnection flare "events" (Kawaguchi et al. 2000), with $\tau_{\text {char }}$ possibly attributed to accretion-disk timescales, e.g., thermal, sound, or viscous.

Early long look, i.e., days, EXOSAT X-ray observations proved useful for estimating PDS on account of their well sampled large amplitude, rapid variations. Their PDS are featureless and "red," $P(f) \propto f^{-\alpha}$ with $\alpha \sim 1.6$, on timescales of minutes to days (Green, McHardy, \& Lehto 1993; Lawrence \& Papadakis 1993), typically providing lower limits on $\tau_{\text {char }}$ due to insufficient sampling of lowfrequency variations. Papadakis \& McHardy (1995) present evidence for $\tau_{\text {char }} \sim 14$ days in NGC 4151 . A recent analysis of XTE data on NGC 3516 by Edelson \& Nandra (1999) presents evidence for $\tau_{\text {char }} \sim 30$ days. The rapid X-ray variations are probably not reconcilable with starburst models, but consistent with flaring activity in a disk corona (Haardt \& Maraschi 1991, 1993). It remains unclear as to whether the measured characteristic timescales may be associated with a physical timescale, because of the small number statistics and dominant measurement uncertainties.

Constructing power spectra from UV and optical observations is more challenging due, mostly, to a combination of smaller amplitude variations on short, $\sim$ day, timescales and, at least for ground-based observations, inhomogeneous unevenly sampled data. Giveon et al. (1999) found similar power spectra with $\alpha=2.0 \pm 0.6$ on timescales of 100-1000 days, for a sample of PG quasars. Cid Fernandes, Sodré, \& Vieira da Silva (2000) found evidence for charac- 
teristic variability timescales of $\sim 600$ days for the same sample. This is notably longer than the above $\tau_{\text {char }}$ derived from X-ray data, and it remains unclear as to whether the physical processes responsible for the X-ray and optical variations are different on timescales of days and years. We may test this hypothesis through higher quality measurements of the UV/optical PDS on these timescales (to be compared with theoretical models) and investigating the possible existence of any $\tau_{\text {char }}$ on timescales less than 100 days.

This paper measures the power density spectra of 13 Seyfert 1 galaxies, through a structure function analysis. The data was obtained by the International AGN Watch ${ }^{2}$ and Ohio State University (OSU; Peterson et al. 1999) and represents a comprehensive sample of UV/optical observations spanning $\sim$ months to years with temporal resolutions of $\sim 1-10$ days. The data allow us to quantify the PDS and possible existence of any $\tau_{\text {char }}$ on timescales less than 100 days, and thereby constrain the origin and ubiquity of the energy-generating mechanisms. In $\S 2$ we discuss the precepts of a structure function analysis. Our results are presented in $\S 3$, and quantified through detailed simulations in $\S 4$. In $\S 5$ we discuss the implications of our results, and conclude with a summary in $\S 6$.

\section{STRUCTURE FUNCTION ANALYSIS}

The method of structure function analysis and its application to astronomical time series is discussed by, e.g., Simonetti, Cordes, \& Heeschen (1985), Hughes, Aller, \& Aller (1992), Press, Rybicki, \& Hewitt (1992), Kawaguchi et al. (1998), and Paltani (1999). A structure function measures the distribution of power over timescales for which the variations are correlated, and is theoretically equivalent to a power-spectrum analysis. The structure function method has the critical advantage of analyzing the times series in the time domain. It partially minimizes windowing and aliasing problems, accentuated by irregularly sampled astronomical data, that plague a Fourier-based power-spectrum analysis. A first-order structure function, for a series of flux measurements $f\left(t_{i}\right), i=1,2,3, \ldots$ is defined by

$$
S(\tau)=\frac{1}{N(\tau)} \sum_{i<j}\left[f\left(t_{i}\right)-f\left(t_{j}\right)\right]^{2},
$$

where the sum is over all pairs for which $t_{j}-t_{i}=\tau$, and $N(\tau)$ is the number of pairs.

For an infinite, stationary random process the structure function is simply related to its autocorrelation function, $\operatorname{ACF}(\tau)$, i.e.,

$$
S(\tau)=2\left[\sigma^{2}-\mathrm{ACF}(\tau)\right],
$$

with $\sigma^{2}$ the variance of the time series. The WienerKhinchin theorem states that the autocorrelation function and power-spectrum are Fourier pairs, thereby structure functions for given power-density spectra (PDS) may be derived. The structure function for the one-sided PDS $P(f)$ is, after Paltani (1999),

$$
S(\tau)=2\left[\int_{0}^{\infty} P(f) d f-\int_{0}^{\infty} P(f) \cos (2 \pi f \tau) d f\right] .
$$

Typically, structure functions are characterized by flat sections with amplitudes of twice the signal variance, $2 \sigma^{2}$, and twice the noise variance, $2 \sigma_{\text {noise, }}^{2}$ on long and short

\footnotetext{
${ }^{2}$ http://www.astronomy.ohio-state.edu/ agnwatch/.
}

timescales, $\tau_{\max }$ and $\tau_{\min }$, respectively. These two flat plateaus are connected by a power-law section on intermediate timescales, i.e., $\tau_{\min } \lesssim \tau \lesssim \tau_{\max }$. This power-law section defines the range of timescales over which the variations are correlated, and its slope depends on the physical mechanism responsible for the intrinsic variations, e.g., shot, flicker noise, etc. The timescale $\tau_{\text {char }} \sim \tau_{\max }$ at which the structure function flattens may represent a robust, characteristic timescale determined by fundamental source characteristics, e.g., mass and size; provided $\tau_{\text {char }} \ll T$, the duration of the monitoring campaign.

It is important to note that observed structure functions (§ 3) will typically deviate from their expected theoretical shape, particularly on longer timescales for which the meansquared variations are of order $2 \sigma^{2}$, on account of nonstationary and finite duration effects (to be discussed more in $\S 4)$. For example; a linear trend in the data will artificially steepen the structure function on intermediate to long timescales, and for a time series with approximately equal fluxes on the longest timescales, the structure function will show a rapid decrease. An understanding of these effects is required in order to assess whether observed features in structure functions are real.

Previous analyses of large optical QSO samples (including hundreds of QSOs) have, typically, constructed ensemble first-order structure functions due to the limited number of epochs and relatively poor, irregular sampling. These structure functions have shown evidence for a steep rise at $\tau<2-3 \mathrm{yr}$ before flattening on longer timescales and suggest characteristic variability timescales in the range of 0.9-2.4 yr (e.g., Hook et al. 1994; Trevese et al. 1994; Cristiani et al. 1996). Hughes et al. (1992) present individual radio structure functions for about 50 radio-loud AGNs, and find mean characteristic variability timescales of 2.0-2.4 $\mathrm{yr}$ with a linear logarithmic rise in structure functions on shorter timescales. More recently, Cid Fernandes et al. (2000) presented a structure function analysis for a sample of PG quasars, finding characteristic variability timescales of $\sim 0.5-3 \mathrm{yr}$.

\section{THE UV/OPTICAL STRUCTURE FUNCTIONS}

We use UV and optical data from five and 12 AGNs, respectively, representing a comprehensive sample of monitoring observations spanning months to years with temporal resolutions of $\sim 1-10$ days. However, we note that our sample is in no way complete. The majority of sources were chosen because they were known or suspected to be reliably variable on timescales of interest; most may be classified as radio-quiet Seyfert 1 galaxies, but 3C 390.3 is a broad-line radio galaxy, and, e.g., NGC 7469 is a starburst Seyfert 1 galaxy. We utilize UV/optical light curves, at approximately 1300 and $5100 \AA$, respectively, from AGN Watch and OSU monitoring programs (§ 1$)$.

We determine binned structure functions through equation (1). The bin midpoints are given by $\tau_{i}=\left(i-\frac{1}{2}\right) \delta$ for $i=1,2, \ldots,(T / \delta+1)$ with $\delta$ the resolution of the structure function and $T$ the total duration of the light curve. From each bin we subtract $2\left\langle\sigma_{\text {noise }}^{2}\right\rangle$, with $\left\langle\sigma_{\text {noise }}^{2}\right\rangle$ the mean variance of the measurement uncertainties, thereby removing their effects from each structure function. We normalize all our structure functions by $\sigma^{2}$, the light curve's variance (corrected for the measurement uncertainties), to facilitate an easy intercomparison; hence all structure functions should asymptote to $\log S \simeq 0.30$ on long timescales. We 
match $\delta$ to about the median temporal sampling of individual light curves. This resolution defines, approximately, the shortest timescale to which the data are sensitive. Furthermore, we only determine the structure function for bins with greater than six data pairs. The statistical uncertainties in our structure functions are defined by $\sigma_{i} /\left(N_{i} / 2\right)^{1 / 2}$, with $\sigma_{i}$ the root mean square (rms) deviations about the mean structure function value in bin $i$ and $N_{i}$ the number of pairs of data points in bin $i$. Our ad hoc, but reasonable, error estimates reflect the fact that not all pairs of measurements in a given bin are independent. This appears acceptable, since the observed structure functions are much smoother than their $\sigma$ values would suggest and not notably inconsistent with their error bars defined by the standard deviation of the mean value for each bin. Finally, for cases where a linear trend clearly contributes to the UV/optical time series, biasing the structure function analysis, we detrend the data by subtracting a linear least-squares fit. This prevents statistical fluctuations in the poorly defined lowfrequency power from biasing our high-frequency power estimates, discussed in detail by Welsh (1999).

\subsection{UV Structure Functions}

The UV light curves may be roughly described by aperiodic fractional flux variations, defined by a rms divided by mean flux of $\sim 15 \%$ on timescales of 1 month, with temporal resolutions of $\sim 0.2-4$ days for $\sim 2-14$ months. The light curves are all IUE observations, except for the HST 1993 campaign on NGC 5548.

Figure 1 presents our binned structure functions for five sources. Typically, they all exhibit the characteristic shape outlined in $\S 2$. We note, by attempting to remove the effects of measurement uncertainties we suppress and/or remove the expected flat plateau on the shortest timescales. For cases where the structure function still notably flattens off, e.g., NGC 5548, this is most likely due to a slight underestimation of the true measurement uncertainties. A power-law section, delineating the variability correlation length on intermediate timescales, is obvious for all sources, except perhaps NGC 3783. In this case there is suggestion of a power-law section on $\tau \leq 10$ days, but it remains ill defined, primarily due to lack of temporal resolution. On longer timescales, the structure functions flatten on timescales of $\sim 6-40$ days before exhibiting oscillations.

Oscillations in $S(\tau)$ occur on timescales for which the mean-squared amplitude variations are of order $2 \sigma^{2}$. On these longer timescales our structure functions are comparatively ill-defined on account of the reduced number of data pairs available. This complicates any detailed analysis. The diversity in character of these oscillations reflects, mostly, the unique character of the individual source variations. The oscillations result from light curves being dominated by one or two large outbursts, finite duration, and nonstationary effects, e.g., disparate flux levels at the start and end of monitoring and/or quasi-periodic oscillations (not well suited to a structure function analysis). Indeed, one visually notes a quasi-periodic variation of $\sim 16$ days in the UV/optical light curves of NGC 7469. This periodicity is confirmed with a classic periodogram analysis, but is only 3 times the length of the monitoring experiment and thereby viewed with caution. Its reality or otherwise should be tested with independent data sustained over many months.
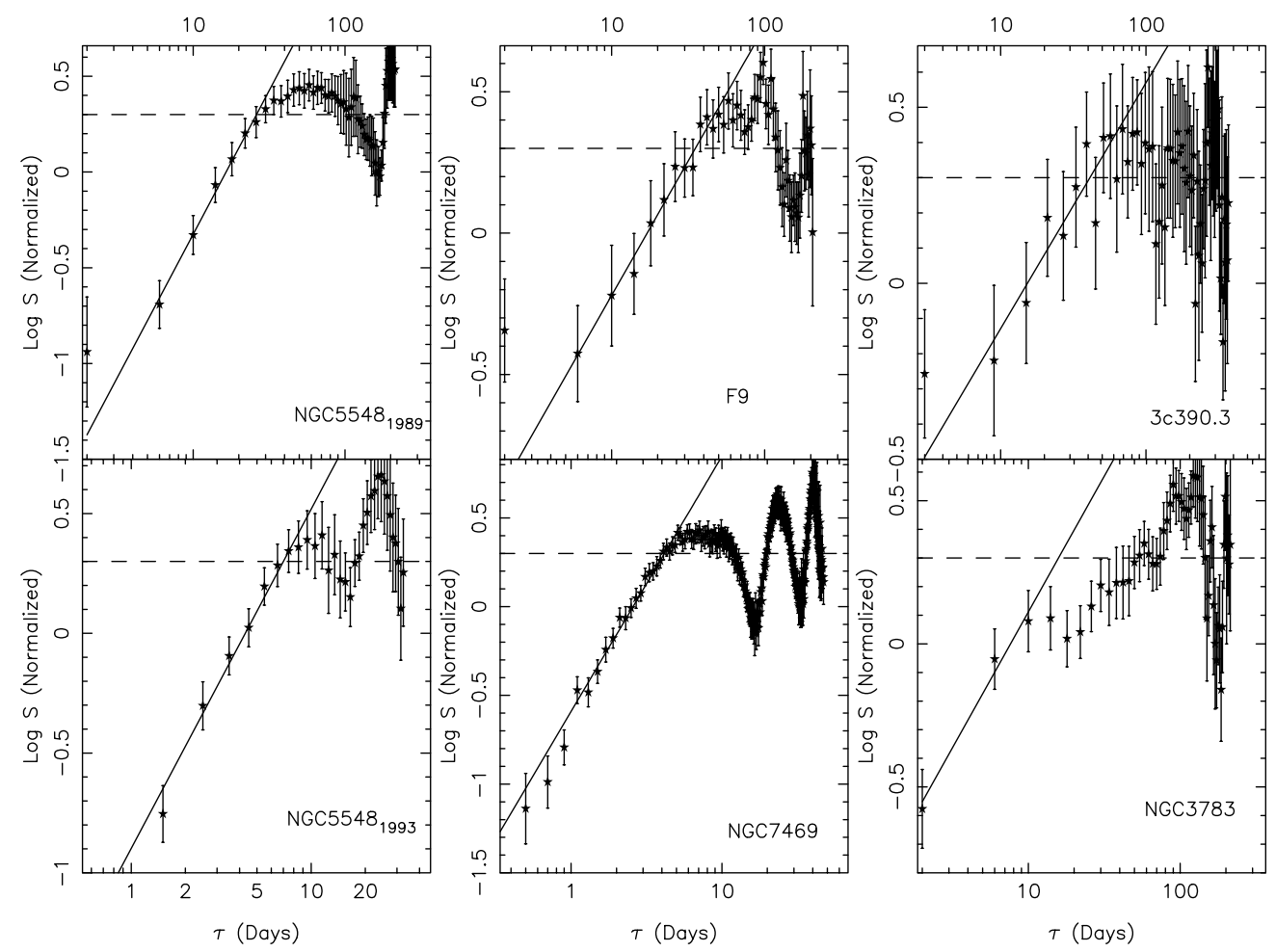

FIG. 1.-UV structure functions for five active galaxies, as labeled. NGC 5548 appears twice since it has been the target of two intensive monitoring campaigns in 1989 and 1993. The structure functions are normalized by their light-curve variance, and the effects of measurement uncertainties have been removed (§3). A full discussion is given in $\S 3.1$. 
Any features in our structure functions on these long timescales should be viewed with caution for the above reasons.

The power-law slope of the structure functions depends on the physical mechanism responsible for the intrinsic variations. We quantify this slope by fitting the model $\log S=b \log \tau+a$ to the appropriate range of timescales, $\tau_{\min } \leq \tau \leq \tau_{\max }$, with $\tau_{\min }$ and $\tau_{\max }$ determined by visual inspection. Results are detailed in Table 1. Column (1) details the AGN (NGC 5548 appears twice, since it has been the target of two intensive monitoring campaigns in 1989 and 1993), columns (2) and (3) give the total monitoring duration $T$ and structure function resolution $\delta$, columns (4) and (5) detail the $\tau_{\min }$ and $\tau_{\max }$ timescales between which the above model is fitted, column (6) gives the number of data points included in the fit, column (7) the reduced $\chi_{v}^{2}$, and column (8) the slope $b$. In all cases, over the range of timescales defined in Table 1, a power-law representation is acceptable. The fact our typical reduced $\chi_{v}^{2} \sim 0.3$ may suggest we have overestimated the errors on our structure functions. However, this is most likely a negligible effect, since the uncertainty in our power-law slopes is dominated by nontrivial systematic effects to be discussed in $\S 4$.

We find a range of power-law slopes of 0.70-1.50, with an unweighted mean slope of $b=1.16 \pm 0.14$. The quoted uncertainty denotes the error in the mean slope, i.e., the standard deviation of measurements about the mean value divided by the square root of the number of measurements. It is worth noting, for the independent 1989 and 1993 NGC 5548 monitoring campaigns, our respective slope measurements of $b=1.50 \pm 0.19$ and $b=1.42 \pm 0.14$ are in good agreement.

It has been noted by a number of authors (e.g., Hufnagel \& Bregman 1992; Kawaguchi et al. 1998) that for a stationary time series there exists a simple relationship between the above structure function power-law slope and the powerdensity spectrum slope, i.e., $\alpha=b+1$ with $P(f) \propto f^{-\alpha}$. However, Paltani (1999) notes this is only true in the limit of infinite time series, otherwise some bias is introduced (see eq. [3]). If the structure function samples the true underlying variations adequately, these biases will be minimized, and the above relationship will give a good first-order approximation for $\alpha$. The smoothness of the UV/optical variations on timescales of days (Welsh et al. 1998; Edelson et al. 2000), coupled with prior estimates of their PDS $\left[P(f) \propto f^{-\alpha}\right.$ with $\alpha \sim 2$; e.g., Krolik et al. 1991 and Reichert et al. 1994], suggest we are not grossly undersampling or otherwise the true variations for our best-sampled light curves. This being the case, it is reasonable to utilize the above approximation to infer first-order estimates of $\alpha$. This suggests a mean UV PDS with $\alpha=2.16 \pm 0.14$ (see $\S 5$ ). In $\S 4$, we take the first empirical steps in quantifying the magnitude of this bias, and show it is of order $\sim 10 \%$ for the data herein.

\subsection{Optical Structure Functions}

The optical light curves for the AGN Watch campaigns (i.e., NGC 5548, NGC 3783, NGC 7469, 3C 390.3, and NGC 4151) may be roughly characterized by aperiodic fractional flux variations of $\sim 5 \%$ on timescales of 1 month, with temporal resolutions of $\sim 1-4$ days for $\sim 2-14$ months. For the other, OSU-monitored sources, the temporal resolution is typically $\sim 10$ days for $\sim 6$ years.

Figure 2 presents our binned structure functions for 12 sources. Our structure function for Fairall 9 is not presented here, since it is dominated by noise. Furthermore, we only consider optical data from the 1989 monitoring campaign on NGC 5548; as labeled in Figure 2. In $\S 3.3$, we consider additional observations of NGC 5548 to establish the validity of observed features in its structure function, discussed therein. We note this methodology is dictated by the strategy of analyzing individual monitoring experiments separately. NGC 5548 is a special case, since it is the most intensively monitored AGN and remains the subject of an ongoing monitoring experiment that thus far has lasted $\sim 11$ yr. The structure functions are similar to those presented in $\S 3.1$, displaying a power-law section and oscillatory behavior on intermediate and long timescales, respectively. We quantify the power-law slope in the same way as before, $\S 3.1$, and present our results in Table 2 . The column headings are identical to those of Table 1 . In all cases a power-law representation over a range of timescales is appropriate, with reduced $\chi_{v}^{2} \sim 0.4$.

The range of power-law slopes is $0.84-1.56$, with an unweighted mean slope of $b=1.11 \pm 0.06$. As before, the quoted error is the error in the mean slope. Both the range of observed slopes and mean value are consistent with those derived from our UV structure functions of $\S 3.1$. This suggests the underlying physical mechanism(s) responsible for the UV/optical variability are identical and will be further discussed in $\S 5$. We note the power-law slopes for objects with both UV and optical observations are consistent to within the estimated uncertainties. As discussed in $\S 3.1$, it is reasonable to utilize the naive approximation, $\alpha=b+1$, to infer first-order estimates of $\alpha$ from our derived power-law

TABLE 1

UV STRUCTURE FUNCTIONS

\begin{tabular}{|c|c|c|c|c|c|c|c|}
\hline $\begin{array}{l}\text { Source } \\
\text { (1) }\end{array}$ & $\begin{array}{c}T \\
\text { (days) } \\
(2)\end{array}$ & $\begin{array}{c}\delta \\
\text { (days) } \\
(3)\end{array}$ & $\begin{array}{c}\tau_{\min } \\
\text { (days) } \\
\text { (4) }\end{array}$ & $\begin{array}{c}\tau_{\max } \\
\text { (days) } \\
\text { (5) }\end{array}$ & $\begin{array}{l}N \\
\text { (6) }\end{array}$ & $\begin{array}{l}\chi_{v}^{2} \\
\text { (7) }\end{array}$ & $\begin{array}{c}b \\
(8)\end{array}$ \\
\hline NGC $3783 \ldots \ldots$. & 222 & 4 & 2 & 10 & 3 & 0.31 & $0.95 \pm 0.25$ \\
\hline NGC $7469 \ldots \ldots$ & 48 & 0.2 & 0.4 & 5 & 23 & 0.36 & $1.44 \pm 0.06$ \\
\hline NGC $5548^{\mathrm{a}} \ldots$ & 39 & 1 & 1.5 & 10 & 9 & 0.35 & $1.42 \pm 0.14$ \\
\hline NGC $5548^{b} \ldots \ldots$ & 235 & 4 & 3 & 28 & 6 & 0.12 & $1.50 \pm 0.19$ \\
\hline Fairall $9^{c} \ldots$ & 240 & 4 & 6 & 50 & 12 & 0.14 & $0.95 \pm 0.15$ \\
\hline $3 \mathrm{C} 390.3^{\mathrm{c}} \ldots \ldots \ldots$ & 429 & 6 & 9 & 71 & 11 & 0.26 & $0.70 \pm 0.22$ \\
\hline
\end{tabular}

${ }^{\text {a }}$ Data taken from the 1993 monitoring campaign.

${ }^{\mathrm{b}}$ Data taken from the 1989 monitoring campaign.

${ }^{\mathrm{c}}$ Denote light curves for which a linear trend has been removed $(\S 3)$. 

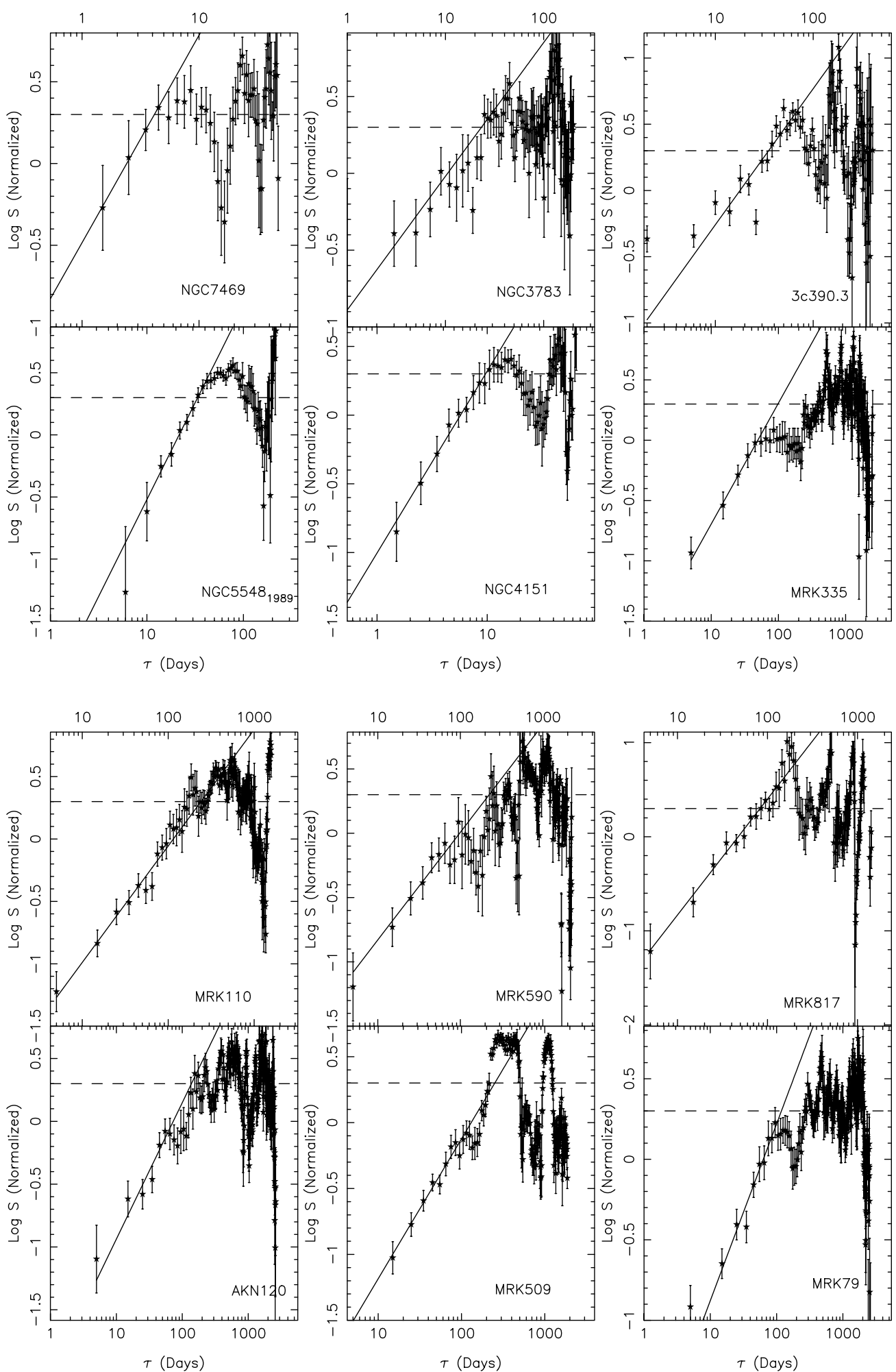

Fig. 2.-Optical structure functions for 12 active galaxies, as labeled. The structure functions are normalized by their light curves variance, and the effects of measurement uncertainties have been removed ( 3 ). A full discussion is given in $\S 3.2$.

slopes. This suggests a mean optical PDS with $\alpha=2.11 \pm 0.06$ (see $\S 5$ and $\S 4)$.

\subsection{Characteristic UV/Optical Timescales $\tau_{\text {char }}$}

The majority of structure functions in Figure 1 flatten on timescales of $\sim 6-40$ days for which the variations are of order $\sigma$. For NGC 3783, the power-law section is tentatively defined, and the structure function appears to flatten off before the nominal $\sigma$ level. We estimate, more objectively, the characteristic timescales $\tau_{\text {char }}$ at which the flattening occurs by fitting theoretical structure functions, derived from symmetric triangular flare profiles (Cid Fer- 
TABLE 2

OpticAl StruCture Functions

\begin{tabular}{|c|c|c|c|c|c|c|c|}
\hline $\begin{array}{l}\text { Source } \\
\text { (1) }\end{array}$ & $\begin{array}{c}T \\
\text { (days) } \\
(2)\end{array}$ & $\begin{array}{c}\delta \\
\text { (days) } \\
(3)\end{array}$ & $\begin{array}{c}\tau_{\min } \\
\text { (days) } \\
\text { (4) }\end{array}$ & $\begin{array}{c}\tau_{\max } \\
\text { (days) } \\
(5)\end{array}$ & $\begin{array}{l}N \\
(6)\end{array}$ & $\begin{array}{l}\chi_{v}^{2} \\
(7)\end{array}$ & $\begin{array}{c}b \\
(8)\end{array}$ \\
\hline NGC $3783 \ldots \ldots$. & 226 & 2 & 3 & 10 & 4 & 0.41 & $0.87 \pm 0.53$ \\
\hline NGC $7469 \ldots \ldots$. & 58 & 1 & 1 & 5 & 4 & 0.00 & $1.27 \pm 0.58$ \\
\hline NGC $5548^{a} \ldots \ldots$ & 237 & 4 & 8 & 40 & 8 & 0.14 & $1.56 \pm 0.18$ \\
\hline 3 C $390.3^{b} \ldots$ & 551 & 4 & 9 & 71 & 16 & 2.35 & $1.03 \pm 0.09$ \\
\hline NGC $4151^{b}$. & 99 & 1 & 1.5 & 10 & 9 & 0.13 & $1.32 \pm 0.22$ \\
\hline Akn $120^{\mathrm{b}} \ldots$ & 2864 & 10 & 10 & 63 & 5 & 0.97 & $1.09 \pm 0.27$ \\
\hline Mrk $110^{b}$. & 1737 & 10 & 10 & 50 & 4 & 0.10 & $0.93 \pm 0.28$ \\
\hline Mrk $335 \ldots \ldots \ldots$ & 2600 & 10 & 10 & 63 & 5 & 0.15 & $1.00 \pm 0.23$ \\
\hline Mrk $509 \ldots \ldots \ldots$ & 1908 & 10 & 10 & 100 & 9 & 0.49 & $1.06 \pm 0.14$ \\
\hline Mrk $590 \ldots \ldots \ldots$ & 2551 & 10 & 10 & 100 & 9 & 0.44 & $0.84 \pm 0.20$ \\
\hline Mrk $79^{b} \ldots \ldots \ldots$ & 2829 & 10 & 10 & 100 & 9 & 0.41 & $1.10 \pm 0.13$ \\
\hline Mrk $817^{b} \ldots \ldots \ldots$ & 1493 & 10 & 10 & 100 & 9 & 0.29 & $1.23 \pm 0.16$ \\
\hline
\end{tabular}

${ }^{\text {a }}$ Data taken from the 1989 monitoring campaign.

${ }^{b}$ Denote light curves for which a linear trend has been removed $(\S 3)$.

nandes et al. 2000), to appropriate ranges of timescales. Their functional form is defined in Appendix B, equation (B6) of Cid Fernandes et al. (2000). We define $\tau_{\text {char }}$ to be equal to the flare lifetime. We note our choice of flare profile is largely arbitrary, guided by the applicability of generalized Poissionian models to AGN variability (Cid Fernandes et al. 2000). Our adopted method is merely intended to give zero-order estimates for $\tau_{\text {char }}$, since the flattening occurs over a limited range of timescales and is typically poorly defined. The systematic uncertainties in our $\tau_{\text {char }}$ estimates may be larger $(\S 4)$. Results are given in Table 3. Columns (1) and (2) detail the source and data type, columns (3) and (4) give $\tau_{\min }$ and $\tau_{\max }$ timescales included in the fit, and column (5) gives $\tau_{\text {char }}$ (equivalent to the flare lifetime) and its 95\% confidence limits. The confidence limits are determined by permitting fits for which $\chi^{2} \leq(N-2)+6.17$, with $N$ the number of data points. For NGC 5548 (1989 campaign), NGC 7469, Fairall 9, and NGC 3783 we find $\tau_{\text {char }} \sim 40,6,42$, and 7 days, respectively. The structure function for the 1993 NGC 5548 monitoring campaign flattens off on a timescale of $\sim 10$ days. This is the result (\$4) of the finite duration, 39 days, of the monitoring experiment and may not be associated with a $\tau_{\text {char }}$; hence is

TABLE 3

Characteristic UV/Optical VARIABILITY TimesCales

\begin{tabular}{|c|c|c|c|c|}
\hline $\begin{array}{l}\text { Source } \\
\text { (1) }\end{array}$ & $\begin{array}{c}\text { Data } \\
\text { (2) }\end{array}$ & $\begin{array}{c}\tau_{\min } \\
\text { (days) } \\
\text { (3) }\end{array}$ & $\begin{array}{c}\tau_{\max } \\
\text { (days) } \\
\text { (4) }\end{array}$ & $\begin{array}{r}\tau_{\text {char }} \\
\text { (days) } \\
(5)\end{array}$ \\
\hline NGC $3783 \ldots \ldots$ & UV & 2 & 25 & $7_{-3}^{+8}$ \\
\hline NGC $7469 \ldots . .$. & UV & 0.4 & 10 & $6_{-1}^{+1}$ \\
\hline NGC $5548^{\mathrm{a}} \ldots \ldots$ & UV & 3 & 80 & $40_{-12}^{+18}$ \\
\hline Fairall $9 \ldots \ldots . .$. & UV & 6 & 90 & $42_{-17}^{+28}$ \\
\hline NGC $7469 \ldots .$. & Optical & 1 & 12 & $5_{-3}^{+7}$ \\
\hline NGC $5548^{a} \ldots \ldots$ & Optical & 8 & 70 & $59_{-12}^{+20}$ \\
\hline NGC $4151 \ldots \ldots$ & Optical & 1.5 & 17 & $13_{-5}^{+11}$ \\
\hline Akn $120 \ldots \ldots \ldots$ & Optical & 10 & 110 & $67_{-25}^{+35}$ \\
\hline Mrk $335 \ldots \ldots \ldots$ & Optical & 10 & 200 & $49_{-23}^{+31}$ \\
\hline Mrk 509 ......... & Optical & 10 & 150 & $93_{-27}^{+33}$ \\
\hline Mrk $590 \ldots \ldots \ldots$ & Optical & 10 & 90 & $51_{-23}^{+59}$ \\
\hline Mrk $79 \ldots \ldots \ldots$ & Optical & 10 & 150 & $94_{-23}^{+29}$ \\
\hline
\end{tabular}

${ }^{a}$ Data taken from 1989 monitoring campaign. not reported as such. There may be a suggestion that $3 \mathrm{C}$ 390.3 's structure function flattens on a timescale of $\sim 80$ days, however, the distinction between its flat section and onset of oscillations is unclear on account of the large measurement uncertainties.

For a number of sources in Figure 2, a similar flattening of the structure function occurs on timescales $\tau_{\text {char }} \sim 5-94$ days for which the amplitude of variations approaches or is of order $\sigma$. Here, the flattening is more poorly defined on account of the noisier, lower amplitude, and less homogeneous optical observations. We estimate $\tau_{\text {char }}$, as before (see Table 3), only for sources with a clearly defined, albeit rather subjectively, flat section before any characteristic oscillations; the flat section should be distinct from any oscillations to within the measurement uncertainties. For the AGN Watch campaigns the structure functions are found to first flatten on timescales $\tau_{\text {char }} \sim 59,5$, and 13 days for NGC 5548, NGC 7469, and NGC 4151, respectively. As above, there may be a suggestion that 3C 390.3's structure function flattens on a timescale of $\sim 80$ days, but this is not well defined. For OSU monitored sources, we find $\tau_{\text {char }} \sim$ 49, 67, 51, 93, and 94 days for Mrk 335, Akn 120, Mrk 590, Mrk 509, and Mrk 79, respectively. Our $\tau_{\text {char }} \sim 13$ days for NGC 4151 agrees well with the Papadakis \& McHardy (1995) estimate of $\sim 14$ days derived from an independent power spectrum analysis of EXOSAT data.

We are uniquely positioned to confirm or otherwise the reality of the observed flattening for NGC 5548, since we have optical spectrophotometric observations with $\sim 4$ day resolution spanning in excess of 11 years for this source (the most recent 3 years remain to be published). Figure 3 presents the optical structure function for 7.2 years of monitoring data on NGC 5548, excluding the 1989 monitoring period; thereby permitting a comparison between the two. The power-law section is clearly defined, $b=1.11 \pm 0.10$, on intermediate timescales defined by $\tau=2-28$ days, and the structure function first flattens on timescales of $34_{-4}^{+3}$ days. The slope may be flatter than that derived from the UV and optical structure functions determined from shorter, specific monitoring experiments. However, the results are reconcilable, at the $2 \sigma$ level, and we conservatively consider them indistinguishable (see Tables 1 and 2); it is possible the errors in our structure function (Fig. 3) are 


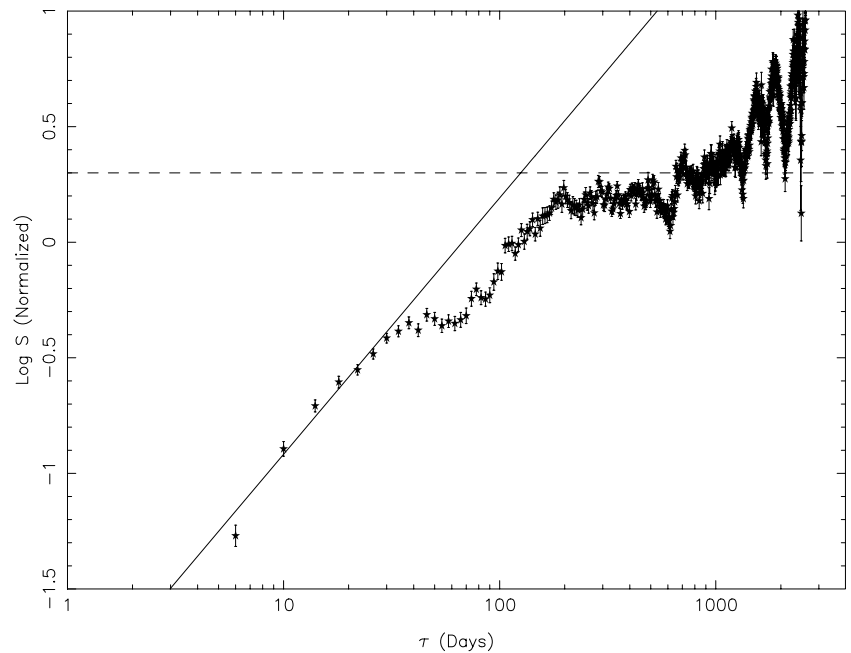

FIG. 3.-The optical structure function for $\approx 7.2$ year spectrophotometric monitoring of NGC 5548, excluding the 1989 monitoring period. This structure function exhibits similar characteristics to those derived from, e.g., UV data, and provides independent evidence for a characteristic variability timescale $\tau_{\text {char }} \sim 40$ days; as evidenced by the point at which the variability power first flattens. See $\S 3.3$ for further discussion.

underestimated and dominated by systematic effects, since for large $N$ it may not be appropriate to assign statistical uncertainties of $\sigma_{i} /\left(N_{i} / 2\right)^{1 / 2}(\S 3)$. Similarly, the flattening of the structure function at a timescale of $\sim 34$ days is indistinguishable from that derived from the aforementioned UV and optical observations at the $2 \sigma$ level (see Table 3). This affords independent evidence supporting the reality of a turnover at $\sim 40$ days in the power distribution of NGC 5548. It is interesting to note possible additional shelves in the structure function of Figure 3 at $\sim 80$ and 200 days. The contrast and definition of these features is entirely questionable. However, one may speculate (as we do in $\S 5$ ) that they represent different $\tau_{\text {chan }}$, i.e., longer timescales possibly related to, e.g., starburst activity.

The above observations may be important if they can be identified as characteristic variability timescales related to fundamental source characteristics. However, as noted, the flattening occurs over a limited range of timescales and is poorly defined. On the longest timescales real structure functions may exhibit complex, oscillatory behavior for a variety of reasons. For these reasons we proceed with caution. In $\S 4$ we discuss first simulations to address whether the observed flattening is real. Our simulations suggest the observed flattening, in all cases, is most likely real and not, e.g., an artifact of finite duration and nonstationary effects. The implications of these results are deferred to $\S 5$.

\section{SIMULATIONS}

A structure function analysis is considerably complicated by finite duration effects and to a much lesser extent, at least for our well-sampled AGN Watch data, sampling problems. A finite random process will initially flatten off on a timescale which is related to its duration. If a time series evidences a linear trend, leading to nonstationary effects, this will artificially steepen the structure function, since its contribution scales as $S \propto \tau^{2}$. Furthermore, as discussed in $\S 3$, oscillations in the structure functions for timescales where the amplitude of variations are of order $\sigma$ may result directly from the character of individual variations. That is, different monitoring experiments display different continuum variations, thereby will very likely give different structure functions in this regime even if the underlying physical processes are identical.

The sampling and binning of the structure functions introduces bias. This bias invalidates the simple relationship between the PDS spectral index $\alpha$ and the logarithmic power-law slope $b$, i.e., $\alpha=b+1$ (§ 3.1). Consider a time series with $\alpha=1$, i.e., one with enhanced variability on short ( $\lesssim 1$ day) timescales, cf. $\alpha=2$. With daily sampling of the variations, one underestimates the power on the shortest timescales and this leads to $b>0$. If the structure function samples the true variations well, these biases are small and the above relationship will give a good first-order approximation for $\alpha$. Of course this is not known a priori, but appears to be a reasonable assumption based on the observations (§ 3.1). The related effects of irregular sampling are far less pronounced for a structure function analysis, provided each bin contains sufficient data to accurately define the amplitude of variability (see below).

We attempt to characterize the above finite duration and sampling effects through simulations. We aim to assess (1) whether the observed flattening of structure functions on longer timescales may be identified with a physical characteristic variability timescale $\tau_{\text {char }}$ unrelated to the finite realization of an essentially infinite process, and (2) the correspondence of the power-law slope $b$ to $\alpha$, the PDS index.

Our simulation methodology follows Welsh (1999). We generate 100 parent light curves from a Fourier series with $P \propto f^{-\alpha}$ and random phases. These light curves have duration $T=10 \mathrm{yr}$, are evenly sampled with $d t=1,4$, or 10 days, and have the continuum variability characteristics listed in Table 1 of Welsh (1999), i.e., appropriately represent the optical variability of the best monitored AGN, NGC 5548. From each parent light curve, we select a number of subsections of prespecified length. This process results in 1000 simulated light curves of a given length. This simulation methodology mimics reality in ensuring the autocorrelation function $\operatorname{ACF}(\tau)$ will be different for each simulated light curve even though the underlying generating mechanism is identical.

For each simulation set of 1000 light curves, defined by $\alpha$ and $T$, we determine the unweighted mean structure function. Figure 4 presents our results for $\alpha=2$ and $T=50$, 100 , and 200 days: Referring to the top panel, the simulated structure functions exhibit much of the behavior detailed in § 3. In particular, the amplitude of variations first reach $\approx \sigma$ for timescales which are approximately a constant fraction of the monitoring duration $T$, i.e., $\tau / T \approx 0.3$, prior to which the structure functions are essentially power laws with no clear intermediate flat section. The bottom panel gives the logarithmic $\mathrm{rms} \sigma_{\mathrm{S}}$ about the mean structure functions. On the shortest timescales, $\tau \lesssim 4$ days, the structure functions estimates are dominated by noise, and may be safely disregarded. The longest timescales are poorly defined on account of poorer statistics, and ambiguous oscillations occur (after the amplitude of variations first reach the $\sigma$ level) due mostly to variations in the autocorrelation functions of individual realizations. This latter effect is also responsible for the significant $\sim 0.2 \mathrm{dex}$ rms deviations on intermediate timescales. This implies that measurement of 


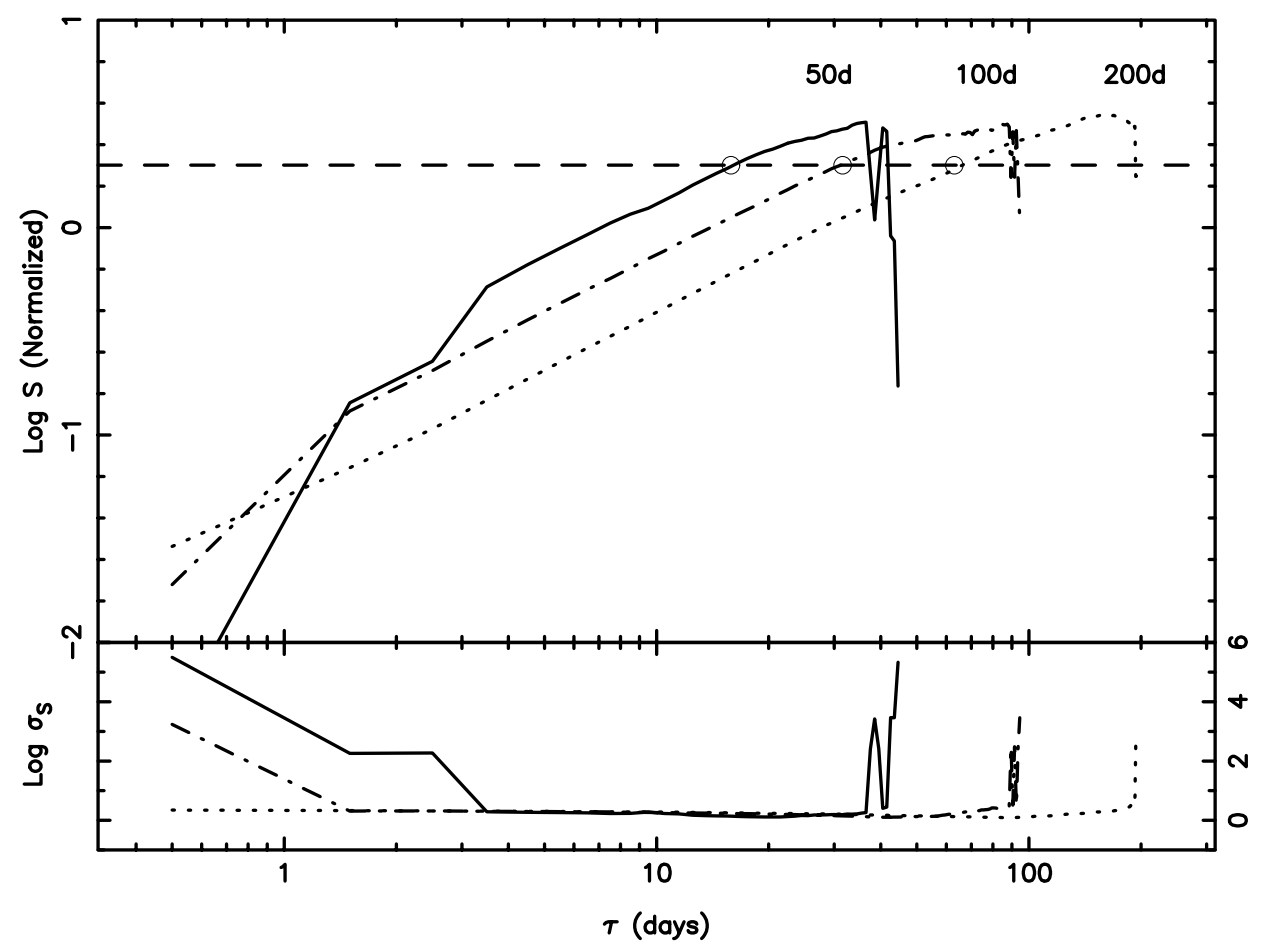

Fig. 4.-Top panel: Simulated mean structure functions for $P(f) \propto f^{-2}$ and $T=50,100$, and 200 days, as labeled. The amplitude of variations first reach $\sigma$ (the effective rms) for timescales $\tau$ which are about a constant fraction of the monitoring duration $T$, i.e., $\tau / T \approx 0.3$; indicated by the open circles for each structure function. On the shortest and longest timescales the structure function estimates are dominated by noise and poor statistics/individual light-curve characteristics, respectively. This is shown by the logarithmic rms $\sigma_{\mathrm{S}}$ detailed in the bottom panel. A full discussion is given in $\S 4$.

the underlying PDS from the finite realization of this stochastic process will be dominated by systematic rather than statistical uncertainties.

Typically, we may reasonably expect a power-law structure function for timescales $\lesssim 0.3 T$ from $P \propto f^{-\alpha}$ variations. However, this may not always be the case given the large uncertainties (Fig. 4, bottom panel) induced mostly by nonstationary effects. For each simulated light curve, we fit a power law to timescales in the range $3 \delta / 2 \leq \tau \leq 0.3 T$, from which follows a $\chi^{2}$ distribution for each simulation set. The median $\chi^{2}$ of this distribution defines a typical structure function, and is indeed reasonably fit by a power law. We estimate the probability of detecting non-power-law structure at $95 \%$ confidence, through counting the number of realizations for which $\chi^{2}>\chi_{\text {median }}^{2}+2 \sigma$, with $\sigma$ the rms deviation about $\chi_{\text {median }}^{2}$. We find, for a wide range of simulation parameters $(T=50,100$, and 200 days; $d t=1,4$, and 10 days), a $\sim 6 \%$ probability of detecting non-power-law structure for timescales $\tau \lesssim 0.3 T$. This probability increases to about $15 \%$ at $68 \%$ confidence. Individual realizations exhibiting non-power-law structure similar to that observed in $\S 3$, i.e., a distinct flattening followed by a subsequent rise or fall, typically fall in the $\gtrsim 2 \sigma$ wings of the $\chi^{2}$ distribution.

The timescale $\tau$ for which the amplitude of variations first reaches $\approx \sigma$ depends slightly on the power-density spectrum adopted and is essentially independent of resolution; for $\alpha=1-3, \tau / T \approx 0.2-0.4$. The smaller values of $\alpha$ lead to shallower structure function power-law sections, and as detailed below and in $\S \S 3.1$ and 3.2, this is not observed. We may therefore reasonably conclude that for the observed structure functions $(\S 3)$ that show significant flattening on timescales $\tau<0.3 T$, it is most likely real at about $94 \%$ confidence, and represents a characteristic timescale determined by the source characteristics. The flattening of the UV 1993 NGC 5548 structure function occurs at $\tau \approx 0.3 T$ with $T=39$ days and may therefore be attributed to finite duration effects.

The power-law slopes for the structure functions of Figure 4 are essentially identical, with $b=0.88$. This slope naively suggests $(\S 3.1)$ a power-density spectrum index $\alpha=1.88$. This underestimates the true index $\alpha=2$ by $6 \%$. The magnitude of the bias increases to $12 \%$, in the same sense, for evenly sampled simulated data at 10 day resolution. However the derived slopes are consistent to within the considerable uncertainties of approximately \pm 0.3 , resulting from different $\operatorname{ACF}(\tau)$ s for each simulated light curve.

We carried out a series of simulations, similar to those above, for light curves of fixed duration $T=200$ days and variable $\alpha$ and resolution $d t$. For $d t=1$ day, we find our naive power-density spectral indices are biased by $\sim+22 \%,-1 \%,-6 \%,-6 \%$, and $-13 \%$ for $\alpha=1,1.5$, $2,2.5$, and 3 , respectively. The positive and negative signs denote over and underestimation of the true $\alpha$. These biases become, for $d t=10$ day, $\sim+23 \%,-1 \%,-10 \%,-16 \%$ and $>-18 \%$ for $\alpha=1, \quad 1.5, \quad 2, \quad 2.5, \quad$ and 3 , respectively.

For $\alpha=1$, the large bias results from undersampling the rapid variations on the short timescales, thereby underestimating the true power. For larger values, i.e., $\alpha=3$, it stems from overestimating the power on short to intermediate timescales. These effects are exacerbated by degrading the resolution of the simulations. It is clear that sampling effects considerably complicate the predicted correspondence between $b$ and $\alpha$. 
We assess the effects of irregular sampling through simulations that disregard a fixed fraction of data from each individual realization at random. We find a random rejection of $0 \%-50 \%$ of data from individual realizations (using $\alpha=2$ ) has a negligible effect on the aforementioned bias levels and probabilities of detecting spurious structure for timescales $\tau \lesssim 0.3 T$. This confirms our expectation that a structure function analysis is less susceptible to ill effects due to irregular sampling than, say, Fourier methods. A complete treatment would simulate the exact sampling characteristics of each data set. This is beyond the scope of this paper, and would very likely provide no further insight. Instead, we adopt a plausible estimate of $10 \%$ for the magnitude of the systematic error by which we have underestimated our inferred values of $\alpha$. This error is the mean percentage bias derived from our $d t=1$ and 10 day simulations for $\alpha=2$ and 2.5, since our UV/optical structure functions (§ 3) suggest a mean power-density spectrum slope of $\alpha=2-2.5(\operatorname{see} \S 5)$.

\section{DISCUSSION}

A structure function analysis is equivalent to a powerspectrum analysis. The power-law component of the structure function delineates the range of timescales for which the variations are correlated, and its slope may be related to the PDS index through $\alpha=b+1$ in the limit of infinite stationary time series; with $P \propto f^{-\alpha}$ and $S \propto \tau^{b}$ (§ 3.1). In $\S 4$ we showed this is an acceptable first-order approximation for our UV/optical variability data and demonstrated we underestimate $\alpha$ by $\sim 10 \%$; this includes both sampling and finite-duration effects.

The UV/optical structure functions of 13 AGNs all display coherent variability over a range of timescales, as detailed in Tables 1 and 2, and Figures 1 and 2. The unweighted mean UV and optical power-law slopes are $b=1.16 \pm 0.14$ and $b=1.11 \pm 0.06$, respectively. The consistency of the equivalent UV and optical PDS may be good evidence for a common generation mechanism. The global UV/optical unweighted PDS index is $\alpha=2.13$ $\pm 0.06\left({ }_{-0.06}^{+0.22}\right)$; the error in parentheses includes our aforementioned estimated systematic uncertainty (§ 4). This measurement affords a robust determination of the UV/optical PDS for a sample of Seyfert 1 galaxies.

Kawaguchi et al. (1998) calculated artificial structure functions via Monte Carlo simulations for both starburst and SOC disk instability models. These models predict different PDS indices, i.e., $\alpha \sim 2.6$ and 1.9, respectively. Our results perhaps compare more favorably with the latter estimates for disk instability models, since starburst models have difficulty in explaining observed short timescale ( days) UV/optical variability. This may be alleviated by further consideration of thermal instabilities within the compact supernova remnant (Terlevich et al. 1995; Plewa 1995). A definitive statement requires accurately quantifying the range of permitted PDS indices for individual models.

As discussed in $\S 1$, the short timescale PDS derived from X-ray observations may be described by $\alpha \sim 1.6$ (e.g., Lawrence \& Papadakis 1993). The statistical uncertainty in this value is of order \pm 0.2 . We may not conclude with confidence the X-ray and UV/optical PDS are different on these timescales. Indeed, for the four sources we have in common with the samples of Green et al. (1993) and Lawrence \& Papadakis (1993), the X-ray and UV/optical PDS indices are typically in agreement to within the $1 \sigma$ measure- ment uncertainties. The similarity between the X-ray through optical PDS on timescales of days may suggest a common origin. Moreover, it broadly supports scenarios whereby X-rays are generated via Compton upscattering of UV photons from the putative accretion disk (Haardt \& Maraschi 1991, 1993), to later radiatively drive optical variations. This strengthens Czerny, SchwarzenbergCzerny, \& Loska's (1999) similiar suggestion based on a PDS analysis of NGC 5548 observations.

There are number of physical timescales associated with any given source. For convienence, we repeat some of the relevant timescales, following Edelson \& Nandra (1999) and Kawaguchi et al. (1998) and references therein. The light crossing time $t_{1 \mathrm{c}}$ is the fastest possible variability timescale for a coherent, isotropically emitting region,

$$
t_{\mathrm{lc}}=0.011\left(M / 10^{7} M_{\odot}\right)\left(R / 10 R_{\mathrm{S}}\right) \text { days },
$$

with $M$ the black hole mass, $R$ the emission distance from the center of mass, and $R_{\mathrm{S}}=2 G M / \mathrm{c}^{2}$ the Schwarzschild radius. An ADAF accretion timescale (Manmoto et al. 1996) $t_{\text {acc }}$ is roughly comparable to the free-fall velocity,

$$
t_{\text {acc }} \geq 160\left(M / 10^{9} M_{\odot}\right)\left(R / 100 R_{\mathrm{S}}\right)^{3 / 2} \text { days . }
$$

The gas orbital timescale $t_{\text {orb }}$ from Kepler's third law

$$
t_{\text {orb }}=0.33\left(M / 10^{7} M_{\odot}\right)\left(R / 10 R_{\mathrm{S}}\right)^{3 / 2} \text { days . }
$$

Finally, the accretion disk thermal timescale is

$$
t_{\mathrm{th}}=5.3(\gamma / 0.01)^{-1}\left(M / 10^{7} M_{\odot}\right)\left(R / 10 R_{\mathrm{S}}\right)^{3 / 2} \text { days, }
$$

with $\gamma$ the standard disk viscosity parameter. All these timescales depend on the mass of the black hole and the emission distance from the center. These timescales may possibly be associated with characteristic variability timescales $\tau_{\text {char }}$.

The observation of predicted "breaks" in AGN power spectra are tentative. Edelson \& Nandra (1999) show the power spectrum of NGC 3516 flattens on a timescale $\tau_{\text {char }} \sim 30$ days, Papadakis \& McHardy (1995) find $\tau_{\text {char }} \sim$ 14 days for NGC 4151, and Czerny et al. (1999) find $\tau_{\text {char }} \sim$ 30 days for NGC 5548. These latter two estimates compare favorably with our estimates of $\tau_{\text {char }} \sim 13$ and 40 days for NGC 4151 and NGC 5548, respectively. No clear consensus has emerged attributing these $\tau_{\text {char }}$ to a given physical timescale.

We find evidence for "breaks" in the PDS of 10 AGNs (§ 3 and $\S 4$ ). Figure 5 presents our attempt at defining a mass-variability timescale relationship. The top and bottom panels present UV and optical data, respectively. The masses derive from a reverberation analysis, Wandel, Peterson, \& Malkan (1999). The solid lines detail representative light crossing, orbital, ADAF accretion (Manmoto et al. 1996), and thermal timescales, as labeled. We assume sources are accreting at 3\% Eddington (Wandel et al. 1999), and derive emission distances assuming blackbody emission from a Shakura \& Sunyaev (1973) thin disk at $1400 \AA$ (top panel) and $5000 \AA$ (bottom panel), respectively. We note $t_{\mathrm{lc}}$ timescales are not shown in the top panel of Figure 5 on account of their small magnitude. For thermal timescales we assume a viscosity parameter equal to 0.01 . We note the representative timescales are uncertain by at least a factor few to 10 . The open square and circle symbols refer to AGN Watch and OSU monitored sources. The solid star symbol (bottom panel) denotes the mean variability timescale and mass derived from a sample of PG quasars (Cid Fernandes 


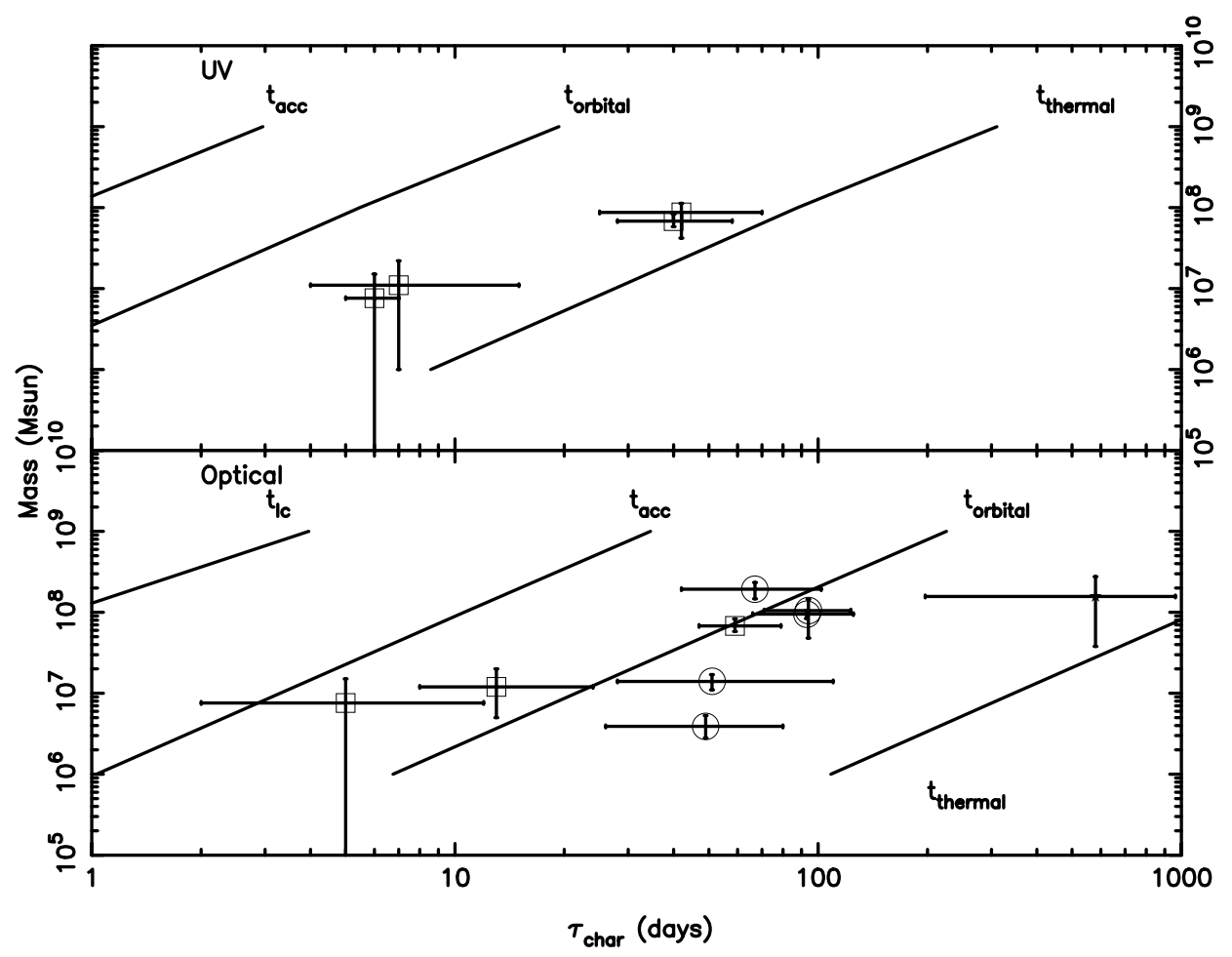

FIG. 5.-Mass-variability timescale relationship derived from our UV (Top panel: § 3.1) and optical (Bottom panel: § 3.2) data. The solid lines detail representative light crossing, orbital, ADAF accretion, and thermal timescales, as labeled. We note light crossing timescales are not shown in the top panel, on account of their small magnitude. There derivation is detailed in $\S 5$. The open square and circle symbols refer to AGN Watch and OSU monitored sources. The solid star symbol (bottom panel) denotes the mean variability timescale and mass derived from a sample of PG quasars (Cid Fernandes et al. 2000).

et al. 2000); the error bars reflect the rms deviations about these quantities.

We grossly confirm theoretical expectations that higher mass systems tend to have longer characteristic variability timescales, and vice versa. The UV timescales appear best represented by orbital or disk thermal timescales. They suggest a viscosity parameter $\gamma \sim 0.03$, consistent with values favored by theoretical and observational results (e.g., Kuraszkiewicz, Loska, \& Czerny 1997 and Laor \& Netzer 1989). At the same time, they are about 2 orders of magnitude longer than the expected light crossing time. This latter timescale defines a scattering region size, assuming the $\mathrm{X}$-rays are generated by Compton upscattering of UV photons from an accretion disk (e.g., Haardt \& Maraschi 1991, 1993). Our results would require significant optical depths of $\sim 15$ in order to smear out the variability and reconcile our observed timescales with $t_{\mathrm{lc}}$; this supports Edelson \& Nandra's similar finding. These suggested large optical depths are a problem for X-ray generation models (e.g., Brainerd \& Lamb 1987).

The optical timescales display larger scatter and may be consistent with, e.g., accretion or orbital timescales. The results are clearly ambiguous on account of large observational uncertainties and the possible existence of different $\tau_{\text {char }}$ to be discussed below. We refer the reader to the bottom panel of Figure 5: The two lower right points denote Mrk 335 and Mrk 590. These sources have similar masses to NGC 7469 and NGC 4151 (which occupy the lower left region) but notably different $\tau_{\text {char }}$ of $\sim 50$ days, compared to 10 days. The resolution of the optical light curves for Mrk 335 and Mrk 590 precludes the measurement of power on timescales of 10 days or less, and we speculate for these cases we are measuring a different variability timescale. In $\S 3.3$ we made similar speculative remarks to explain the longer variability timescales, $\tau_{\text {char }} \sim$ 80 and 200 days, found in NGC 5548. If we plotted these additional timescales on Figure 5, Mrk 335, Mrk 590, NGC 5548, and the PG quasars define a roughly parallel tract to the other datapoints, and provide very tenuous evidence supporting our above speculation. Similarly, it is not possible to attribute these apparently systematically longer timescales to a given physical timescale. They may represent disk thermal timescales, and suggest a viscosity parameter of $\gamma \sim 0.03$. Another intriguing possibility is these longer timescales may be attributable to starburst activity, since the effective lifetime of a compact supernova remnant in the $B$ band is $\tau_{B}^{\mathrm{cSNR}} \approx 75-1400$ days (Aretxaga, Cid Fernandes, \& Terlevich 1997), comparable to the observed range of timescales. Czerny et al. (1999) find evidence for distinct short ( $\$ 30$ days) and long (years) timescale optical variations in NGC 5548, attributing the latter to thermal disk instabilities. It is not inconceivable that more than two distinct physical processes contribute to, at least, the optical variability of AGN on different timescales.

\section{SUMMARY}

We have measured power-density spectra for 13 AGNs through a structure function analysis. We make the following statements:

1. For timescales $\tau \lesssim 60$ days, the mean UV and optical power-density spectra are identical, and may be described by $P \propto f^{-\alpha}$ with $\alpha=2.13_{-0.06}^{+0.22}$. The UV/optical variations may possibly be reproduced by disk instability models. A 
definitive statement will require accurate quantification of the range of permitted PDS power-law indices for plausible theoretical models, e.g., disk instability and starburst. The UV/optical variations are closely related to those at X-ray energies since their PDS indices are essentially indistinguishable.

2. We present evidence for characteristic variability timescales $\tau_{\text {char }} \sim 5-100$ days in 10 AGNs. These timescales possibly reflect dynamical or disk thermal timescales, and are consistent with an accretion disk origin. The association of our derived $\tau_{\text {char }}$ with physical timescales is clearly ambiguous on account of large observational uncertainties. We note there is a significant systematic uncertainty in determining the true, underlying power distribution from the finite realization of an essentially infinite stochastic process. This suggests an upper confidence limit of about $94 \%$ on its measured properties, regardless of the statistical uncertainties.

3 . We find suggestive evidence for multiple optical variability timescales. For masses $M \sim 10^{7} M_{\odot}$, short timescale $(\tau \sim 10$ days) variability may possibly be associated with dynamical timescales, whereas longer timescale $(\tau \sim 50$ days) variability may plausibly be attributed to disk thermal or starburst activity timescales.

This work was supported by NASA grant NAG5-8397. The authors greatly appreciate comments on draft versions of this paper from R. Edelson, R. Cid Fernandes, K. Horne, and K. Korista. The authors thank R. Pogge for useful conversations, and an anonymous referee for useful comments.
Abramowicz, M. A., Bao, G., Lanza, A., \& Zhang, X. 1991, A\&A, 245, 454

Aretxaga, I., Cid Fernandes, R., \& Terlevich, R. 1997, MNRAS, 286, 271

Aretxaga, I., \& Terlevich, R. 1994, MNRAS, 269, 462

Brainerd, J., \& Lamb, F. K. 1987, ApJ, 317, L33

Cid Fernandes, R., Sodré, L., \& Da Silva, L. 2000, ApJ, 544, 123

Cristiani, S., Trentini, S., La Franca, F., Aretxaga, I., Andreani, P., Vio, R., \& Gemmo, A. 1996, A\&A, 306, 395

Czerny, B., Schwarzenberg-Czerny, A., \& Loska, Z. 1999, MNRAS, 303, 148

Edelson, R., et al. 2000, ApJ, 534, 180

Edelson, R., \& Nandra, K. 1999, ApJ, 514, 682

George, I. M., \& Fabian, A. C. 1991, MNRAS, 249, 352

Giveon, U., Maoz, D., Kaspi, S., Netzer, H., \& Smith, P. 1999, MNRAS, 306, 637

Green, A., McHardy, I., \& Lehto, H. 1993, MNRAS, 265, 664

Guilbert, P. W., \& Rees, M. J. 1988, MNRAS, 233, 475

Haardt, F., \& Maraschi, L. 1991, ApJ, 380, L51 1993, ApJ, 413, 507

Hook, I. M., McMahon, R. G., Boyle, B. J., \& Irwin, M. J. 1994, MNRAS, 268,305

Hufnagel, B. R., \& Bregman, J. N. 1992, ApJ, 386, 473

Hughes, P. A., Aller, H. D., \& Aller, M. F. 1992, ApJ, 396, 469

Kawaguchi, T., Mineshige, S., Machida, M., Matsumoto, R., \& Shibata, K. 2000, PASJ, 52, L102

Kawaguchi, T., Mineshige, S., Umemura, M., \& Turner, E. L. 1998, ApJ, 504,671

Krolik, J., Horne, K., Kallman, T., Malkan, M. A., Edelson, R., \& Kriss, G. 1991, ApJ, 371, 541

Kuraszkiewicz, J., Loska, Z., \& Czerny, B. 1997, Acta Astron., 47, 263

Laor, A., \& Netzer, H. 1989, MNRAS, 238, 897

\section{EFERENCES}

Lawrence, A., \& Papadakis, I. 1993, ApJ, 414, L85

Manmoto, T., Takeuchi, M., Mineshige, S., Matsumoto, R., \& Negoro, H. 1996, ApJ, 464, L135

Marshall, H. L., et al. 1997, ApJ, 479, 222

Mineshige, S., Takeuchi, M., \& Nishimori, H 1994, ApJ, 435, L125

Nandra, K., Clavel, J., Edelson, R., George, I., Malkan, M., Mushotzky, R., Peterson, B. M., \& Turner, T. 1998, ApJ, 505, 594

Nandra, K., Le, T., George, I., Edelson, R., Mushotzky, R., Peterson, B. M., \& Turner, T. 2000, ApJ, 544, 734

Paltani, S. 1999, in ASP Conf. 5, BL Lac Phenomenon, ed. L. Takalo \& A. Sillanpää (San Francisco: ASP), 159, 293

Papadakis, I. E., \& McHardy, I. M. 1995, MNRAS, 273, 923

Peterson, B. M., Wanders, I., Bertram, R., Hunley, J., Pogge, R., \& Wagner, R. 1999, ApJ, 511, 513

Peterson, B. M., Wanders, I., Horne, K., Collier, S., Alexander, T., Kaspi, S., \& Maoz, D. 1998, PASP, 110, 660

Plewa, T. 1995, MNRAS, 275, 143

Press, W. H., Rybicki, G. B., \& Hewitt, J. N. 1992, ApJ, 385, 416

Reichert, G., et al. 1994, ApJ, 425, 582

Shakura, N. I., \& Sunyaev, R. A. 1973, A\&A, 24, 337

Simonetti, J. H., Cordes, J. M., \& Heeschen, D. S. 1985, ApJ, 296, 46

Terlevich, R., Tenorio-Tagle, G., Franco, J., \& Melnick, J. 1992, MNRAS, 255,713

. 1995, MNRAS, 272, 198

Trevese, D., Kron, R., Majewski, S., Bershady, M., \& Koo, D. 1994, ApJ, 433, 494

Wandel, A., Peterson, B. M., \& Malkan, M. 1999, ApJ, 526, 579

Welsh, W. 1999, PASP, 111, 1347

Welsh, W., Peterson, B. M., Koratkar, A., \& Korista, K. 1998, ApJ, 509, 118 